Supporting Information

\title{
Discovery, Structure-Activity Relationship and Biological Activity of Histone-Competitive Inhibitors of Histone Acetyltransferases P300/CBP
}

Fangrui Wu, ${ }^{1, \S}$ Yuanda Hua, ${ }^{1, \S}$ Salma Kaochar, ${ }^{2,3}$ Shenyou Nie, ${ }^{l}$ Yi-Lun Lin, ${ }^{1}$ Yuan Yao, ${ }^{1}$ Jingyu Wu, ${ }^{1}$ Xiaowei Wu, ${ }^{1}$ Xiaoyong Fu, ${ }^{4}$ Rachel Schiff, ${ }^{4,5}$ Christel M. Davis, ${ }^{7}$ Matthew Robertson, ${ }^{3}$ Erik A. Ehli, ${ }^{7}$ Cristian Coarfa, ${ }^{3}$ Nicholas Mitsiades, ${ }^{2,3}$ Yongcheng Song ${ }^{* 1,3}$

${ }^{1}$ Department of Pharmacology \& Chemical Biology, ${ }^{2}$ Department of Medicine, ${ }^{3}$ Dan L. Duncan Cancer Center, ${ }^{4}$ Department of Molecular \& Cellular Biology, ${ }^{5}$ Department of Medicine, ${ }^{6}$ Lester and Sue Smith Breast Center, Baylor College of Medicine, 1 Baylor Plaza, Houston, TX 77030, USA.

${ }^{7}$ Avera Institute for Human Genetics, Sioux Falls, SD 57108, USA.

\section{Table of content:}

Chart S1-S3

Figure S1-S4

Representative HPLC traces

${ }^{1} \mathrm{H}$ and ${ }^{13} \mathrm{C}$ NMR spectra for compound 12 Page S12
Page S2-4

Page S5-8

Page S9-11 

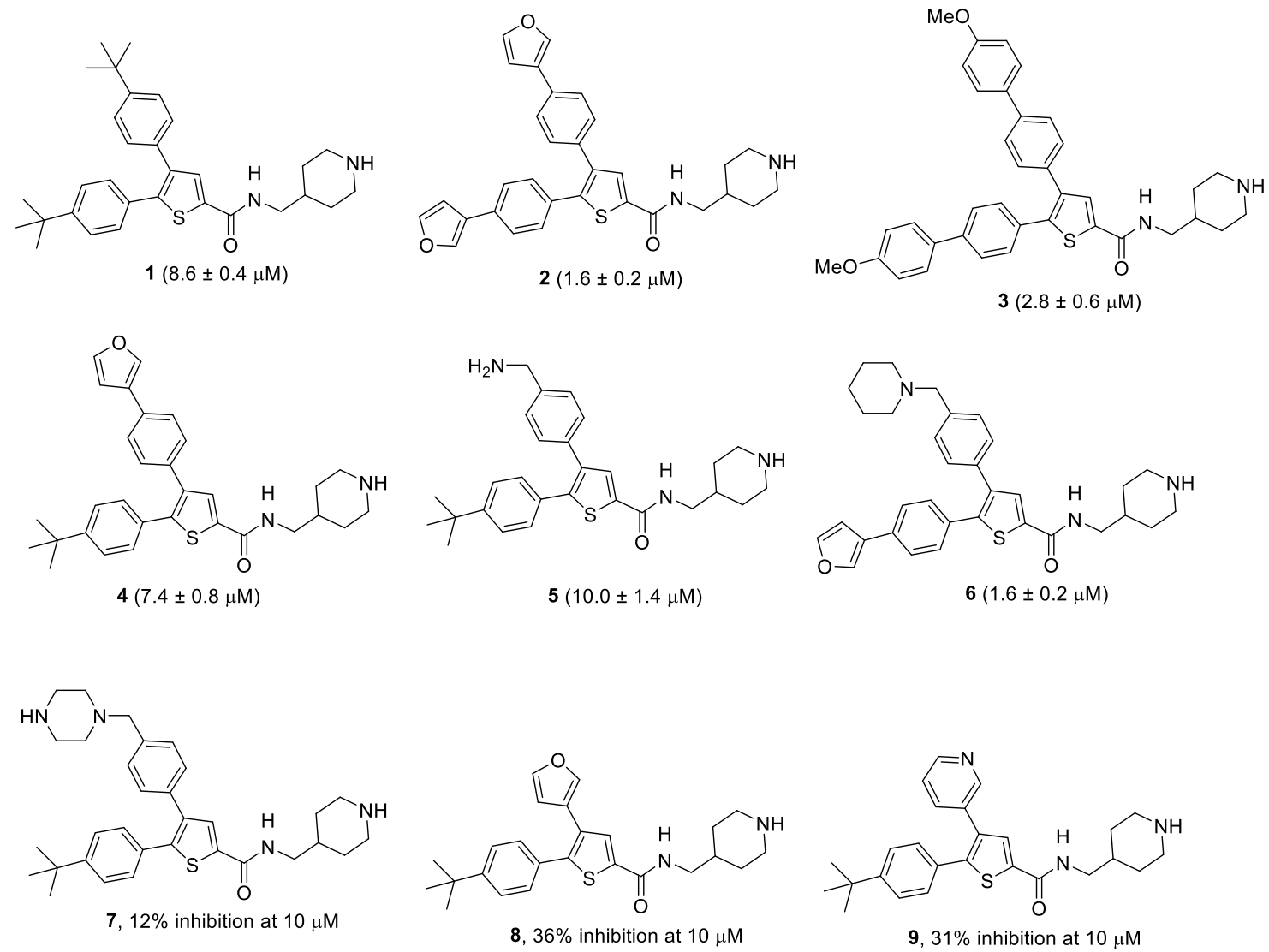

Chart S1. Structures of compounds 1-9 and their inhibitory activities $\left(\mathrm{IC}_{50}\right)$ against p300 HAT. 


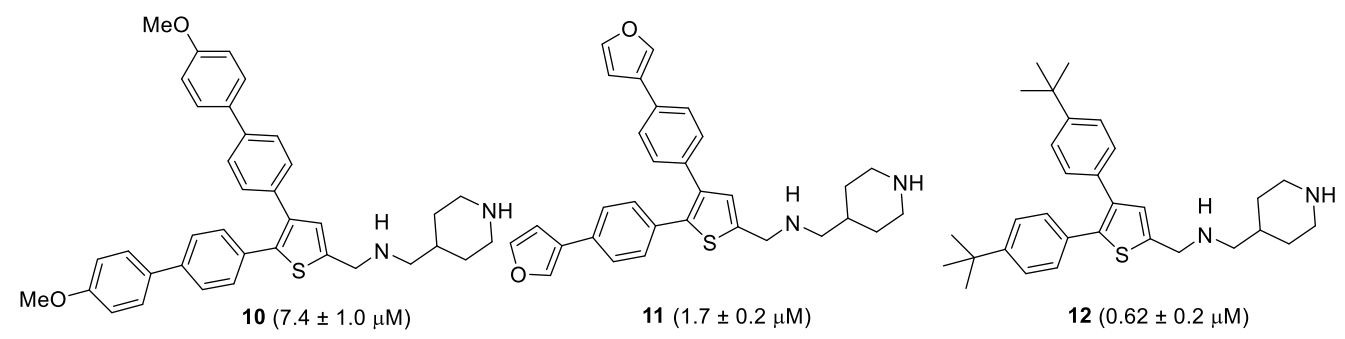

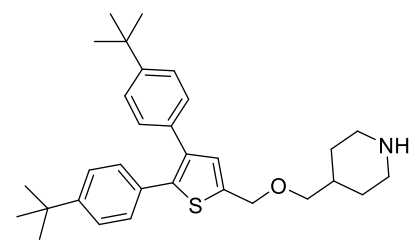

$13,0 \%$ inhibition at $10 \mu \mathrm{M}$

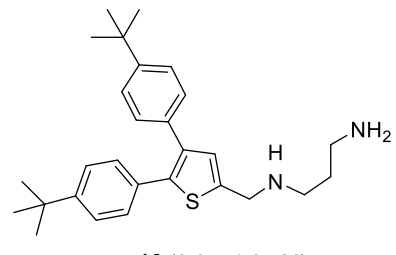

$16(3.0 \pm 1.0 \mu \mathrm{M})$

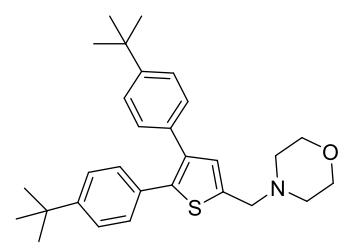

$19,20 \%$ inhibition at $10 \mu \mathrm{M}$

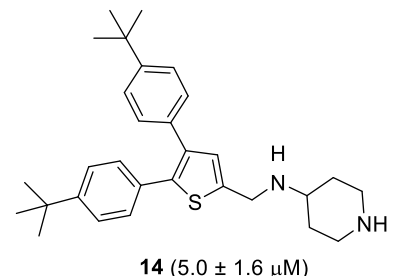

$14(5.0 \pm 1.6 \mu \mathrm{M})$

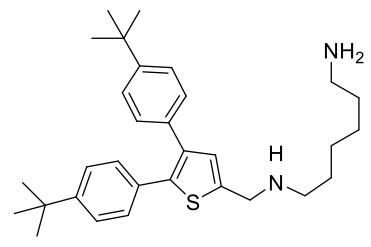

$17(1.4 \pm 0.3 \mu \mathrm{M})$

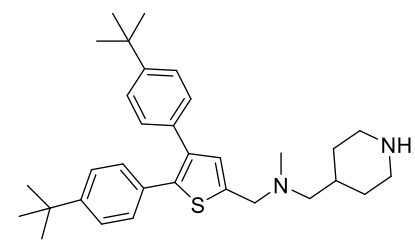

$20(4.4 \pm 1.6 \mu \mathrm{M})$
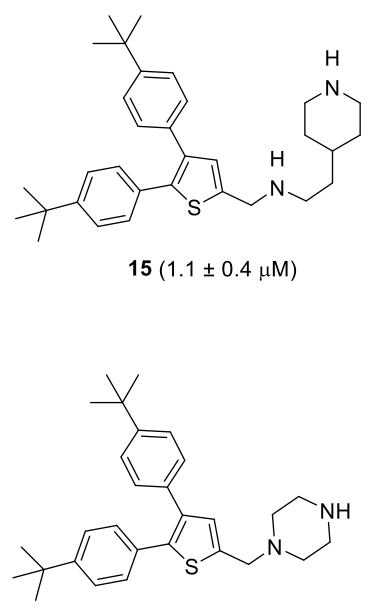

$18,5 \%$ inhibition at $10 \mu \mathrm{M}$

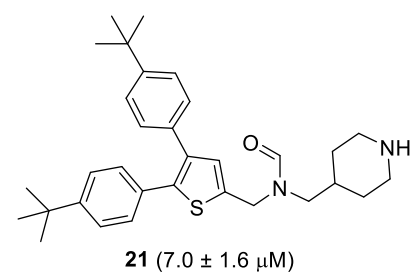

Chart S2. Structures of compounds $\mathbf{1 0 - 2 1}$ and their inhibitory activities $\left(\mathrm{IC}_{50}\right)$ against p300 HAT. 

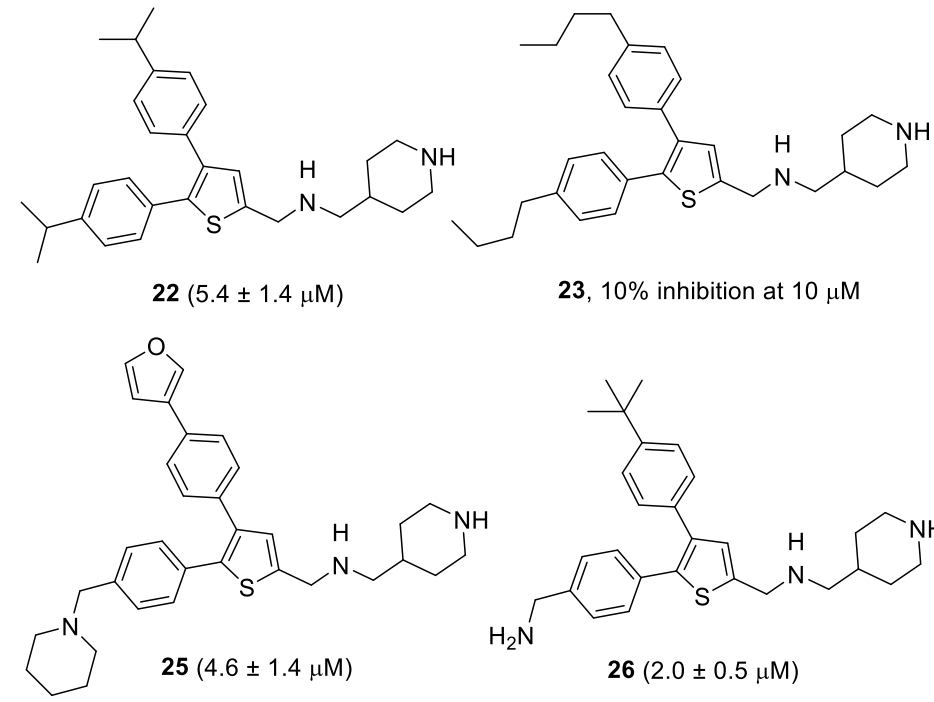

23, $10 \%$ inhibition at $10 \mu \mathrm{M}$
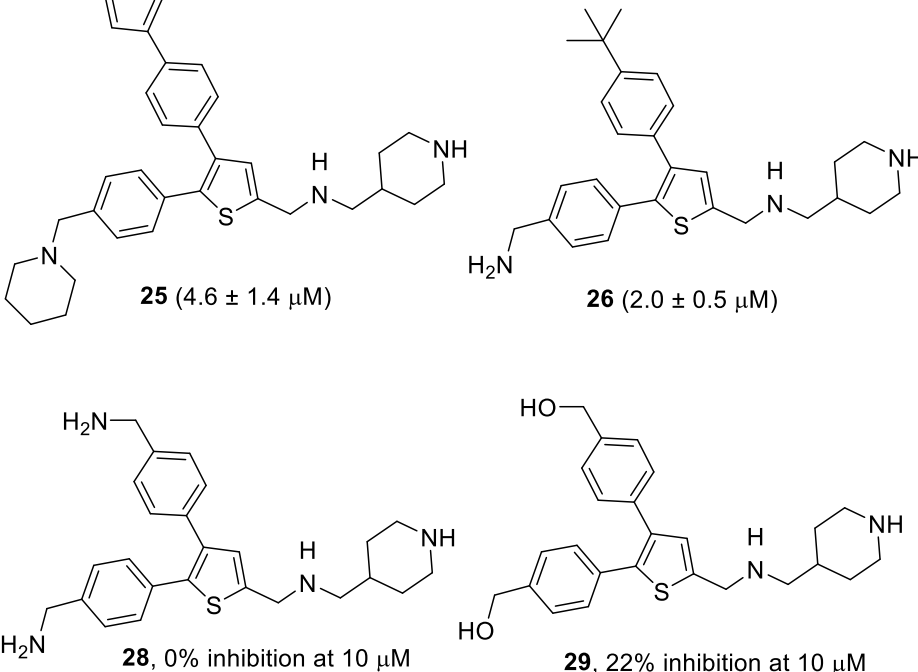

28, $0 \%$ inhibition at $10 \mu \mathrm{M}$

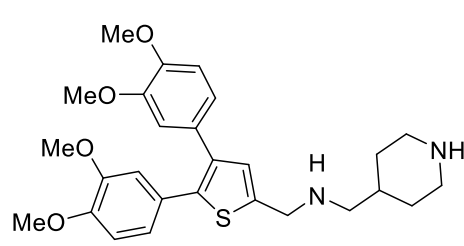

31, $0 \%$ inhibition at $10 \mu \mathrm{M}$

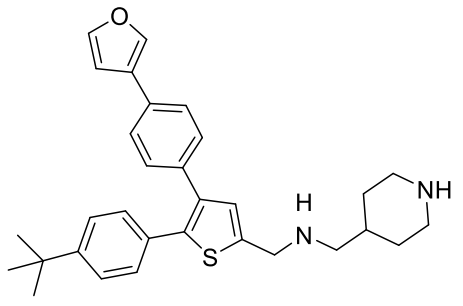

$24(5.8 \pm 0.6 \mu \mathrm{M})$

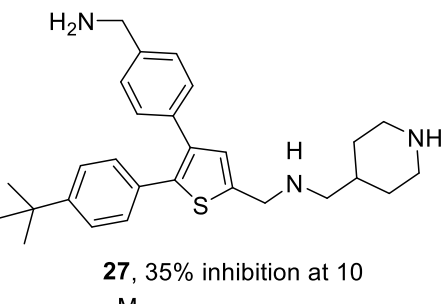

$\mu \mathrm{M}$

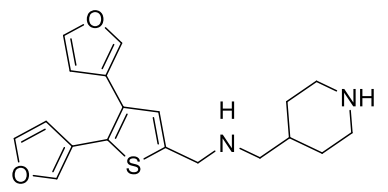

$30,23 \%$ inhibition at 10 $\mu \mathrm{M}$

Chart S3. Structures of compounds $\mathbf{2 2 - 3 2}$ and their inhibitory activities $\left(\mathrm{IC}_{50}\right)$ against p300 HAT. 


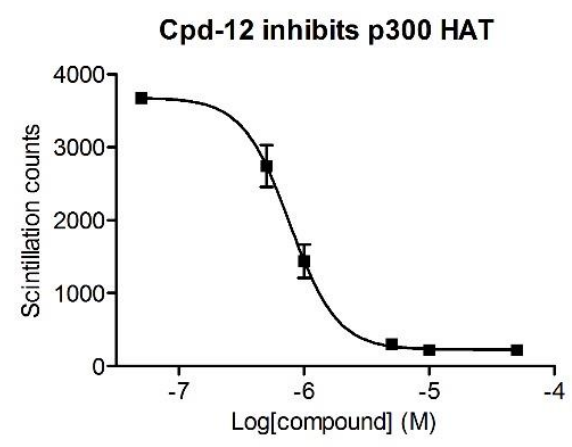

Figure S1. A representative dose response curve of p300 HAT inhibition by compound 12. 


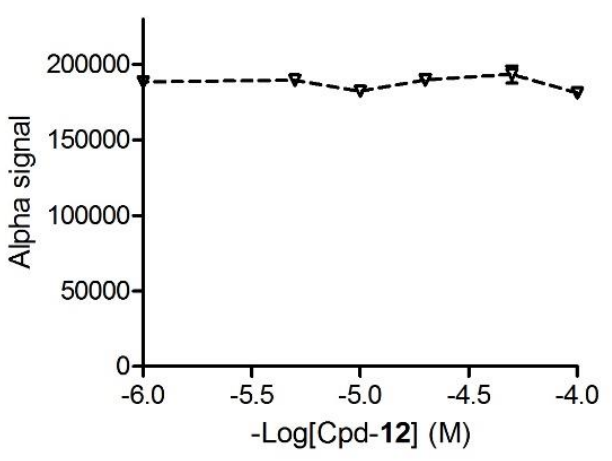

Figure S2. Alpha assay results showing increasing concentrations of compound $\mathbf{1 2}$ did not affect the Alpha-signals using a biotinylated His6 peptide which can directly link the donor and acceptor beads. 

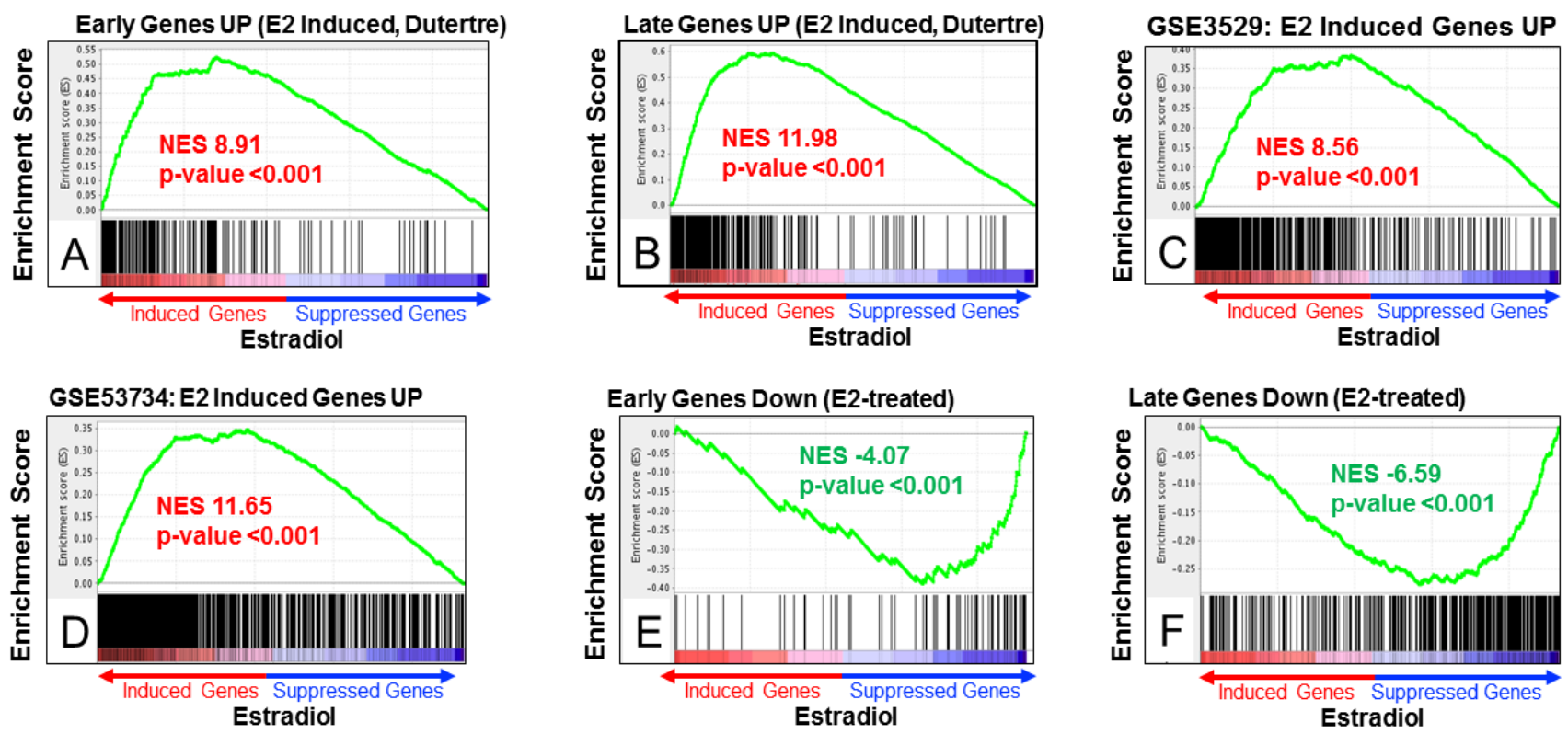

Figure S3. Gene set enrichment analysis (GSEA) results show that supplementation with estradiol caused significant (A-D) upregulation of gene sets that have been reported to be induced by estradiol (from the MSigDB database), and (E, F) downregulation of those suppressed by estradiol. 
Wong:

Embryonic Stem Cell Core

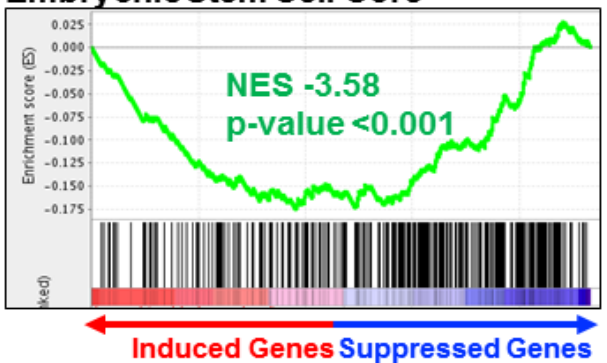

Figure S4. Gene set enrichment analysis (GSEA) shows that treatment of MCF-7 cells with compound 2 significantly suppressed expression of genes known to be involved in maintenance of stem cell state. 
Representative HPLC data

Cpd 12: retention time, $4.204 \mathrm{~min}$; $\mathrm{MS}(\mathrm{M}+\mathrm{H})+$ found 475.3

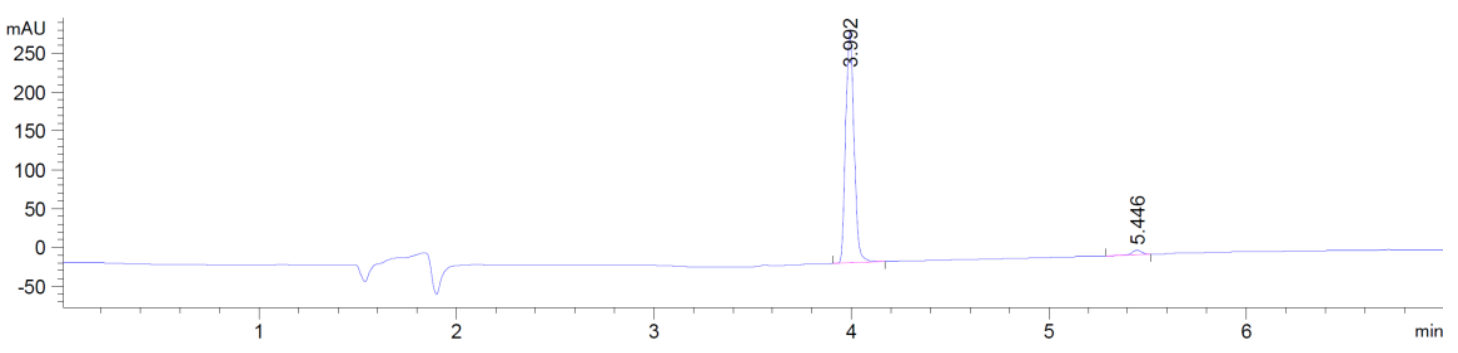

Signal 1: DAD1 A, Sig=254,4 Ref=off

\begin{tabular}{|c|c|c|c|c|c|c|}
\hline $\begin{array}{c}\text { Peak } \\
\quad \#\end{array}$ & $\begin{array}{c}\text { RetTime } \\
\text { [min] }\end{array}$ & Type & $\begin{array}{l}\text { Width } \\
\text { [min] }\end{array}$ & $\begin{array}{c}\text { Area } \\
{\left[\mathrm{mAU}^{*} \mathrm{~s}\right]}\end{array}$ & $\begin{array}{l}\text { Height } \\
{[\mathrm{mAU}]}\end{array}$ & $\begin{array}{c}\text { Area } \\
\%\end{array}$ \\
\hline & & & & --- & -------- & ------ \\
\hline 1 & 3.992 & $\mathrm{BB}$ & 0.0463 & 878.58868 & 299.08826 & 97.7822 \\
\hline 2 & 5.446 & BB & 0.0541 & 19.92757 & 5.80387 & 2.2178 \\
\hline Total & s: & & & 898.51625 & 304.89213 & \\
\hline
\end{tabular}

Cpd 14: retention time, $4.204 \mathrm{~min} ; \mathrm{MS}(\mathrm{M}+\mathrm{H})+$ found 461.3

SYC-1237

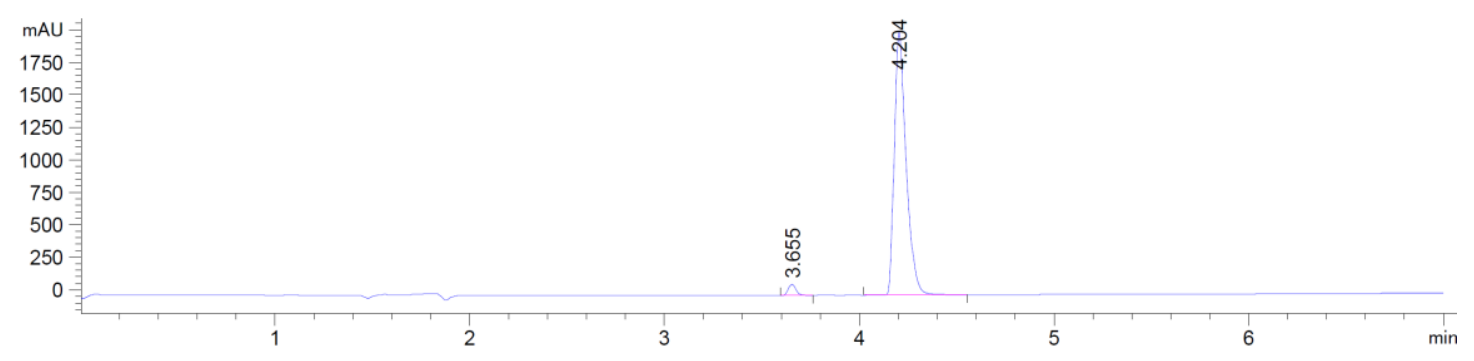


Signal 1: DAD1 A, Sig=254,4 Ref=off

\begin{tabular}{cccccc}
$\begin{array}{c}\text { Peak RetTime Type } \\
\text { \# }\end{array}\left[\begin{array}{c}\text { Width } \\
{[\mathrm{min}]}\end{array}\right.$ & $\begin{array}{c}\text { Area } \\
{[\mathrm{mAU} \text { s }]}\end{array}$ & $\begin{array}{c}\text { Height } \\
{[\mathrm{mAU}]}\end{array}$ & $\begin{array}{c}\text { Area } \\
\%\end{array}$ \\
\hdashline 1 & 3.655 BB & 0.0452 & 244.66147 & 86.02765 & 2.7798 \\
2 & 4.204 BB & 0.0633 & 8556.82031 & 2025.79871 & 97.2202 \\
& & & & & \\
Totals : & & 8801.48178 & 2111.82635 &
\end{tabular}

Cpd 7: retention time, $3.598 \mathrm{~min}$; MS $(\mathrm{M}+\mathrm{H})+$ found 531.3

SYC-1395

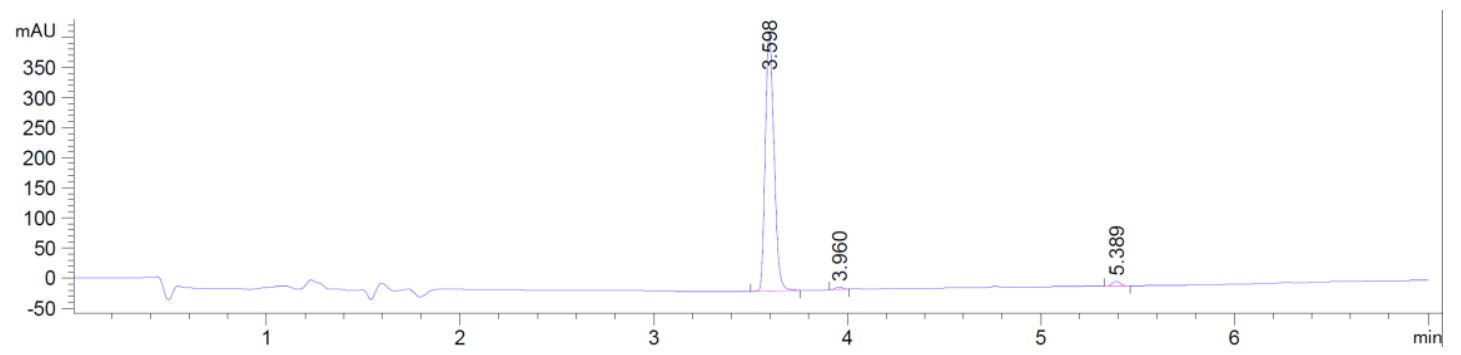

Signal 1: DAD1 A, Sig $=254,4$ Ref $=$ off

\begin{tabular}{|c|c|c|c|c|c|c|}
\hline $\begin{array}{c}\text { Peak } \\
\#\end{array}$ & $\begin{array}{c}\text { RetTime } \\
\text { [min] }\end{array}$ & Type & $\begin{array}{l}\text { Width } \\
\text { [min] }\end{array}$ & $\begin{array}{c}\text { Area } \\
{\left[\mathrm{mAU}^{*} \mathrm{~s}\right]}\end{array}$ & $\begin{array}{l}\text { Height } \\
{[\mathrm{mAU}]}\end{array}$ & $\begin{array}{c}\text { Area } \\
\%\end{array}$ \\
\hline 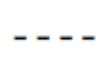 & & & & $\ldots$ & -1 & ---1 \\
\hline 1 & 3.598 & BB & 0.0480 & 1338.31140 & 433.32120 & 97.6114 \\
\hline 2 & 3.960 & BB & 0.0426 & 11.42412 & 4.35543 & 0.8332 \\
\hline 3 & 5.389 & BB & 0.0475 & 21.32511 & 7.43249 & 1.5554 \\
\hline
\end{tabular}

$\begin{array}{lll}\text { Totals : } & 1371.06063 & 445.10912\end{array}$ 
Cpd 33: retention time, 4.292 min; MS (M+H)+ found 554.6

SYC-1478

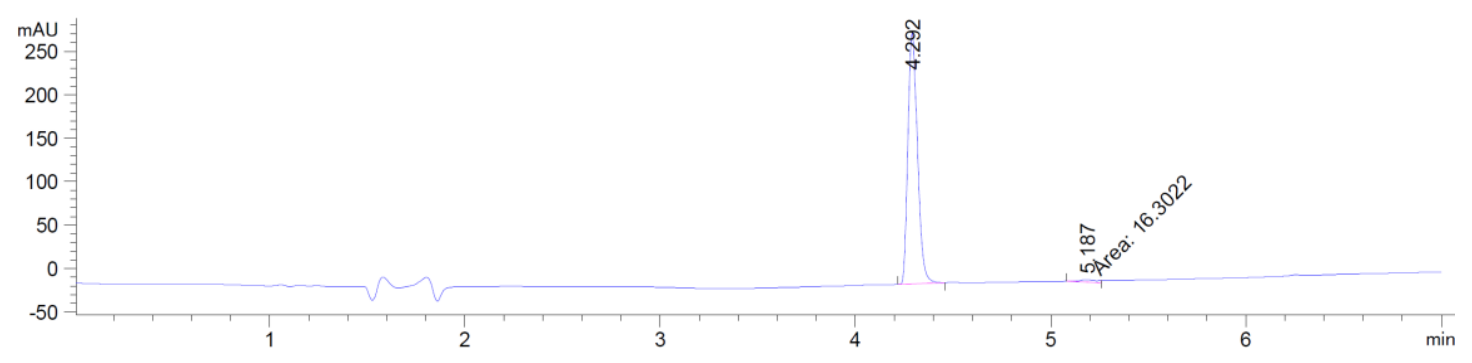

Signal 1: DAD1 A, Sig=254,4 Ref=off

Peak RetTime Type Width Area Height Area

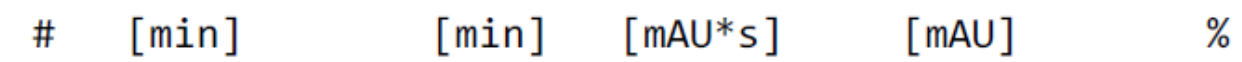

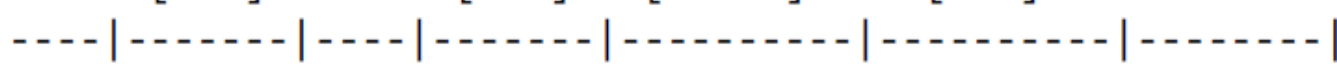

$\begin{array}{lllllll}1 & 4.292 & \text { BВ } & 0.0512 & 977.85840 & 290.90634 & 98.3602\end{array}$

$\begin{array}{llllll}2 & 5.187 \text { MM } & 0.0974 & 16.30218 & 2.79068 & 1.6398\end{array}$

Totals : $\quad 994.16058293 .69703$ 
${ }^{1} \mathrm{H}$ and ${ }^{13} \mathrm{C}$ NMR spectra for compound 12 (next pages) 

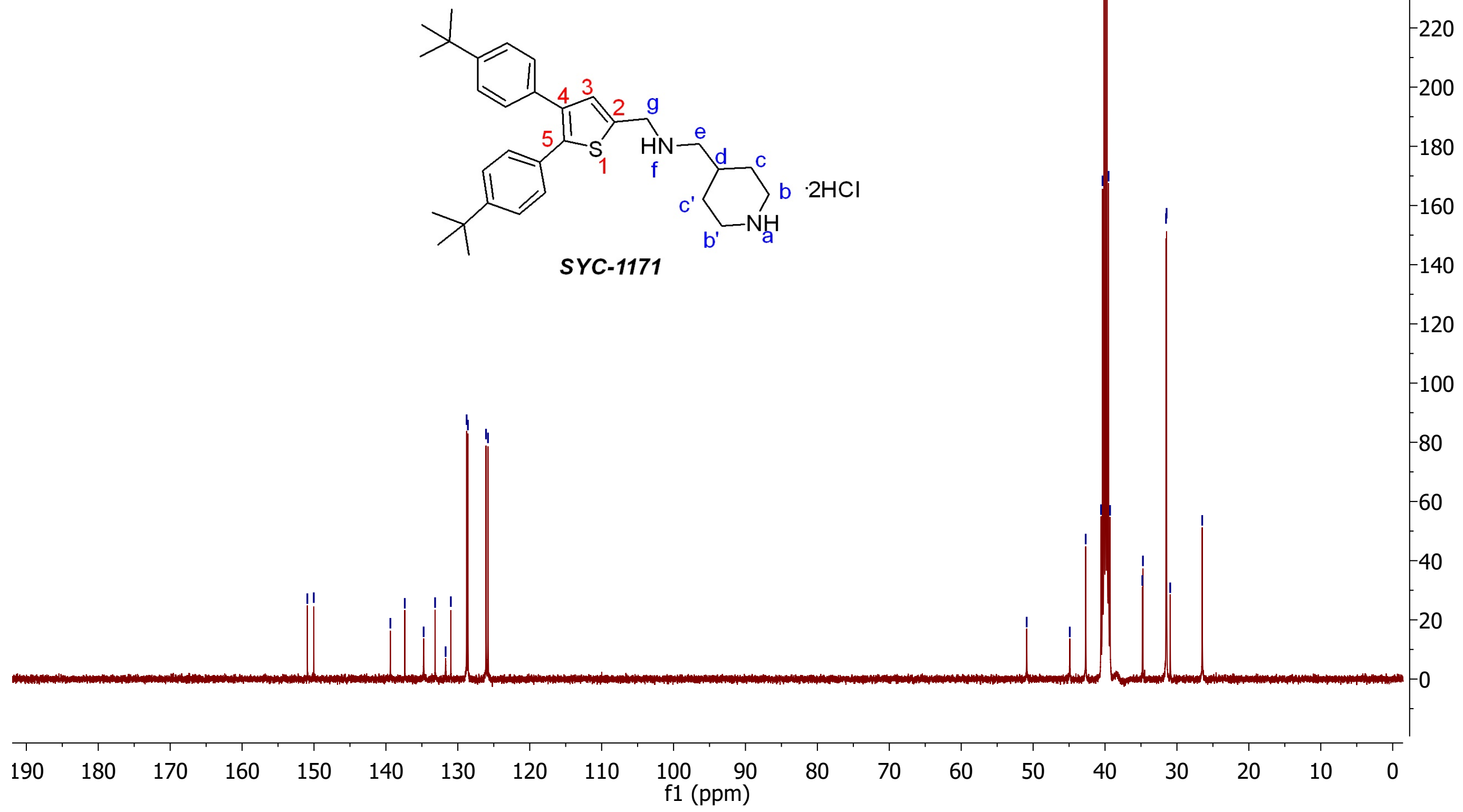